Tropical Journal of Pharmaceutical Research October 2011; 10 (5): 611-618

(C) Pharmacotherapy Group,

Faculty of Pharmacy, University of Benin

Benin City, 300001 Nigeria.

All rights reserved.

Available online at http://www.tjpr.org

Research Article

http://dx.doi.org/10.4314/tjpr.v10i5.10

\title{
Antimalarial Drugs for Pediatrics - Prescribing and Dispensing Practices in a Tanzanian City
}

\section{Appolinary AR Kamuhabwa* and Kunjal Ramji}

Unit of Pharmacology and Therapeutics, School of Pharmacy, Muhimbili University of Health and Allied Sciences,

Dar es Salaam, Tanzania.

\begin{abstract}
Purpose: To assess dispensing and prescribing practices with regard to antimalarial drugs for pediatrics in private pharmacies and public hospitals in Dar es Salaam, Tanzania.

Methods: This was a cross-sectional, descriptive study that assessed the knowledge and practice of 200 drug dispensers in the private community pharmacies in Dar es Salaam. Two hundred (200) parents of children seeking malaria treatment were also interviewed at the public hospitals.

Results: A majority of drug dispensers had moderate (63.3\%) to low knowledge (36.7\%) regarding antimalarial drug use in pediatrics. Parents had moderate (67\%) to low (33\%) level of understanding of the instructions given to them by the prescribers regarding administration of antimalarial drugs to pediatrics at home. Most children were not weighed, and there were indications of wrong calculations of doses of antimalarials in pediatrics.

Conclusion: Overall, there were irrational dispensing and prescribing of antimalarial drugs to pediatrics. Since malaria is a major killer disease in Tanzania, especially in children under five years of age, there is a need to institute measures to ensure rational prescribing, dispensing and use of antimalarial drugs in pediatrics.
\end{abstract}

Keywords: Antimalarials, Dispensing, Knowledge, Pediatrics, Pharmacies, Prescribing 


\section{INTRODUCTION}

Malaria is the leading cause of morbidity and mortality in Tanzania. Every year, 14-18 million new malaria cases are reported in Tanzania, resulting in 120,000 deaths. Of these deaths, 70,000 are in children of less than five years of age. The annual incidence rate is $400-500 / 1,000$ people, and this number doubles for children less than five years of age [1]. It is estimated that over $1 \%$ of GDP is devoted to malaria treatment, representing US $\$ 2.2$ per capita, and $39 \%$ of total health expenditure nationally [2]. Government facilities allocate about one-third of their resources to combat malaria [2]. Private expenditure, primarily on drugs, coils, sprays and bed-nets, represents $71 \%$ of total expenditures.

Studies have shown that irrational prescribing of antimalarial drugs is abundant in most African countries [3-4]. For instance, antimalarials and antibiotics are prescribed together for pediatrics, with little consideration of resistance and adverse drug reaction development [3-4]. Prescription errors are very common, and prescribing analgesics with antimalarial drugs is also a widespread practice. The patient's temperature could be lowered using non drug methods such as fanning and tepid sponging. These methods are particularly important for young children, and can help in bringing down the temperature even without giving antipyretics.

In most places, Tanzania inclusive, the private retail sector has been identified as an important source of drugs close to people's homes $[5,6]$. However, given the dominance of malaria treatment outside government facilities, strategies to control behaviour in the private sector are neccesary. The regimens dispensed by drug dispensing personnel in the private pharmaceutical outlets are often inadequate, with regard to the type of drug and their dosage [7]. Lack of medical awareness and the increasing number of pediatric medicines available over the counter serve to highlight the growing importance of the community pharmacist in ensuring appropriate medicines use [8].

The aim of this study was to assess prescribing and dispensing practices of artemether-lumefantrine to pediatric patients in the public hospitals and private pharmacies. The specific objectives were to assess parents' awareness and knowledge regarding malaria in children as well as their level of understanding of given instructions.

\section{METHODS}

\section{Study area}

Dar es Salaam is the largest and the commercial city in Tanzania. The city has a population of about 4 million people. It is divided into three municipals (Ilala, Kinondoni and Temeke) where the study was conducted. Each municipal has a public hospital which provides care for people living within the municipal. In each municipal, there are also private community pharmacies which provide pharmaceutical care, including selling of antimalarial drugs to the public.

\section{Study population and data collection Drug dispensing personnel from community pharmacies}

A list of currently registered private retail community pharmacies in Dar es Salaam was obtained from Tanzania Food and Drugs Authority (TFDA). By September 2009, there were 233 private retail community pharmacies in Dar es Salaam. Out of these pharmacies, 27 were located in Temeke, 96 in Ilala and 110 in Kinondoni municipals. Two hundred pharmacies were chosen by random sampling using balloting method, and one dispenser in each pharmacy was interviewed.

A pharmacy student in her final year of studies collected data as mystery shopper [910]. She visited pharmacies posing as a guardian of the child who is suffering from malaria. She requested to see the pharmacist 
for advice before she could purchase antimalarial drugs for the child. If the pharmacist was not present, pharmaceutical technicians, pharmaceutical assistants or any other dispensing personnel was requested to provide advice. A designed form was used to get information about drug dispenser's knowledge regarding side effects of antimalarial drugs, antimalarial-drug interactions, dosage of antimalarial drugs according to the weight of a child, and antimalarial medicines proposed by the drug dispensers in comparison to the National Malaria Treatment Guidelines. Immediately after leaving the pharmacy the simulated client filled-in responses from the dispenser in the checklist form.

A knowledge scale was prepared; one point was awarded for each correct answer and a zero point for an incorrect answer. The drug dispenser's knowledge was then graded as low (0-2), medium (3-4) and high (5-6) knowledge based on the correct responses given.

\section{Parents of children seeking malaria treatment at public municipal hospitals}

Two hundred parents of children seeking for malaria treatment were interviewed at the public municipal hospitals in Dar es Salaam. Every consenting parent of the child $(\leq 5$ years) was interviewed at the outpatient clinic after being prescribed antimalarial drugs for his/her child. Questionnaires contained questions regarding child's age, management of malaria-suspected symptoms before visiting the health facility, history of fever during the present illness and use of antimalarial drugs prior to visiting the health facility. Parents were also asked if they received instructions from prescribers on how to administer the drugs to their children at home. They were then asked to repeat the instruction they received from the prescribers about the dose and duration of use for the prescribed antimalarial drugs for the children. Antimalarial drug prescriptions given to parents were checked to assess quality of prescribing in pediatrics.

\section{Prescribers attending to pediatric patients at public municipal hospitals}

Prescribers who had attended to children during the day of interview were interviewed in the afternoon. This was the time when prescribers were available for interviews, as they were less busy compared to the morning hours. A total of 9 prescribers were interviewed at the outpatient pediatric clinics in the municipal hospitals. Questionnaires were designed to obtain information on physical examination of a child before prescribing antimalarial drugs, supportive care for malaria in pediatrics, consideration of the child's weight when prescribing antimalarial drugs, general side effects of antimalarial drugs in pediatrics and prescribing according to the National Malaria Treatment Guidelines.

\section{Data analysis}

The data were analyzed using the Epi Info programme version 3.4. The Chi- Square and P-values were employed to check the significance of study variables. The results were considered significant at $p<0.05$. The sub-questions were equally weighed, with one point for the correct response and zero point for incorrect response. The number of questions relevant to assess the knowledge was totaled. The total numbers were divided by three in order to obtain three categories; low, moderate and high.

\section{Ethical issues}

When using a mystery shopper to assess the knowledge of drug dispensers, participants were not informed in advance and asked for consent to participate in the study. Permission to conduct the study at the selected retail community pharmacies and municipal hospitals was obtained from TFDA and municipal medical officer in-charge, respectively. Informed consent was sought 
from parents of children seeking for malaria treatment, and prescribers at the municipal hospitals. The study was granted ethical clearance from Muhimbili University of Health and Allied Sciences Research and Publications Committee.

\section{RESULTS}

Knowledge of parents about malaria and its management in pediatrics

Table 1 shows the socio-demographic characteristics of the 200 pediatric patients and their parents who were recruited for the study. Out of 200 children who were prescribed antimalarial drugs, 106 (47\%) were males and 94 (53\%) were females. Majority $(69.5 \%)$ of pediatrics were in the age group of 2 years. Most (83\%) parents were relatively young parents, aged between 20 and 29 years. In terms of education, majority (92\%) of parents had attained primary school education.

About a two-thirds $(67 \%)$ of parents had moderate level of understanding the instructions that were given by prescribers regarding the use of antimalarial drugs in children at home. The remaining $(33 \%)$ had low level of understanding the instructions. Only $2(1 \%)$ parents had high level of knowledge regarding supportive care, $69 \%$ had moderate level of knowledge and 30\% had low level of knowledge. In general, older parents had relatively high level of understanding of supportive care measures than younger parents $(p<0.05)$. Supportive care measures that were mentioned by parents included use of oral rehaydration salt $(65.5 \%)$, giving paracetamol to control pain and fever $(57.5 \%)$, use of herbal preparations (14\%) and treatment with cold water to lower body temperature (14\%). Table 2 presents the most common signs and symptoms of malaria in pediatrics as reported by parents.
Table 1: Socio-demographic characteristics of children and their parents $(n=200)$.

\begin{tabular}{lcc}
\hline $\begin{array}{l}\text { Socio-demographic } \\
\text { characteristic }\end{array}$ & Number & Percentage \\
\hline Age of child (years) & $(\mathbf{n})$ & $(\%)$ \\
1 & 20 & 10.0 \\
2 & 139 & 69.5 \\
3 & 18 & 9.0 \\
4 & 8 & 4.0 \\
5 & 15 & 7.5 \\
Gender of children & & \\
$\quad$ Males & 106 & 53.0 \\
$\quad$ Females & 94 & 47.0 \\
Education level of & & \\
parents & & \\
$\quad$ No formal education & 10 & 5.0 \\
$\quad$ Standard seven & 184 & 92.0 \\
$\quad$ Secondary education & 6 & 3.0 \\
Age of parents (years) & 166 & 83.0 \\
$20-29$ & 26 & 13.0 \\
30-39 & 8 & 4.0 \\
$\quad 40-49$ & & \\
\hline
\end{tabular}

Table 2: Reported signs and symptoms of malaria in pediatrics at public hospitals $(n=200)$.

\begin{tabular}{lcc}
\hline Sign/symptom & $\begin{array}{c}\text { Number of } \\
\text { parents (n) }\end{array}$ & $\begin{array}{c}\text { Frequency } \\
(\%)\end{array}$ \\
\hline Diarrhoea & 29 & 14.5 \\
Vomiting & 63 & 31.5 \\
Pallor & 95 & 47.5 \\
Malaise & 127 & 63.5 \\
Fever & 180 & 90 \\
\hline
\end{tabular}

Seventy eight percent of parents indicated that their children were physically examined before they were prescribed with antimalarial drugs. About $60 \%$ of parents were counseled about the importance of ensuring that drugs are given to children at the prescribed doses and duration. Twenty six percent of parents were informed about the possible drug interaction of the prescribed antimalarials with other drugs. Only $7.5 \%$ of parents were advised about the possible side effects of the prescribed antimalarial drugs and the need to seek medical advice if children experienced severe side effects at home. 


\section{Knowledge and dispensing practice of drug dispensers in community pharmacies}

Out of the 200 drug dispensing personnel involved in the study, 60 (30\%) were pharmacists, $20 \quad(10 \%)$ pharmaceutical technicians, $82(41 \%)$ nurse assistants and $38(19 \%)$ sales persons. The knowledge of drug dispensers about good dispensing practice of antimalarial drugs in pediatrics was assessed using a mystery shopper at the retail private community pharmacies. None of the drug dispensers could be categorized as highly knowledgeable about antimalarial drug use in pediatrics. However, pharmaceutical personnel (pharmacists and pharmaceutical technicians) were comparatively more knowledgeable than non-pharmaceutical personnel (nurse assistants and sales persons) $(P=0.0019)$. Table 3 gives a summary of the knowledge levels of drug dispensers regarding antimalarial drug use in pediatrics.

Table 3: Knowledge levels of drug dispensers on the use of antimalarial drugs in pediatrics $(n=200)$.

\begin{tabular}{lcccccccc}
\hline $\begin{array}{l}\text { Level of } \\
\text { knowledge }\end{array}$ & \multicolumn{2}{c}{ Pharmaceutical personnel } & \multicolumn{3}{c}{ Non-pharmaceutical personnel } \\
& Pharmacist & $\begin{array}{c}\text { Pharmaceutical } \\
\text { technician }\end{array}$ & Nurse assistant & Sales person \\
High & $\boldsymbol{n}$ & $\%$ & $\boldsymbol{n}$ & $\%$ & $\boldsymbol{n}$ & $\%$ & $\boldsymbol{n}$ & $\%$ \\
\cline { 2 - 10 } Medium & 0 & 0 & 0 & 0 & 0 & 9 & 0 & 0 \\
Low & 47 & 78.3 & 12 & 60 & 55 & 67.1 & 12 & 31.6 \\
\hline
\end{tabular}

A total of $122(61 \%)$ drug dispensers were willing to dispense antimalarial drugs without a prescription or laboratory results. The remaining $78(39 \%)$ refused to dispense the drugs without a prescription or confirmed malaria diagnosis. For those who were willing to dispense antimalarial drugs without prescription, only 94 (47\%) drug dispensers requested to know the weight of the child before dispensing the drugs.

About $87 \%$ of the visited 200 private retail community pharmacies were stocked with artemether-lumefantrine, which is the first line treatment for uncomplicated malaria in children above $5 \mathrm{~kg}$ body weight in Tanzania. Quinine, which is the first line treatment for complicated malaria and uncomplicated malaria for children less than $5 \mathrm{~kg}$ was stocked in $80 \%$ of the pharmacies. Amodiaquine (72.7\%) and halofantrine (36 $\%)$ were also among the most stocked antimalarial drugs in the pharmacies.

\section{Prescribing practice in public hospitals}

Nine prescribers who were present at the outpatient pediatric clinics during the course of the study were interviewed about prescribing of antimalarial drugs in pediatrics. Eight out of 9 prescribers had moderate knowledge on the precaution, side effects, supportive care and other necessary information that should be noted when prescribing antimalarial drugs to pediatrics. All of the nine prescribers indicated that because of attending many patients it is not possible to weigh all of the children seeking for malaria treatment. Therefore, in most cases prescribers ask the parents the weights of their children when prescribing antimalarial drugs.

The prescription of each child was checked to identify the prescribed antimalarial drugs, dosage and weights of children. A total of 129 $(64.5 \%)$ children were prescribed artemetherlumefantrine and 71 (35.5\%) were prescribed quinine. All the pediatric patients were above $5 \mathrm{~kg}$ of weight. Parents of children who were 
prescribed with quinine were asked if their children had presented with signs and symptoms that were suggestive of complicated or severe malaria. Since these children were not admitted to the wards, and from the explanation of parents about the signs and symptoms presented by the children, it seems that none of these children had severe or complicated malaria to necessitate use of quinine. In addition to antimalarial drugs, some children were also prescribed with co-trimoxazole $(2.5 \%)$, oral rehydration salt (4\%), amoxicillin (7\%) and glucose $(11.5 \%)$. Majority $(75 \%)$ of the children were only prescribed with antimalarial drugs.

\section{DISCUSSION}

In Dar es Salaam, there are over 240 private retail community pharmacies. Availability of artemether-lumefantrine, which is the first line treatment for uncomplicated malaria in children above five kilogram body weight was $87 \%$ in the private retail community pharmacies. Quinine, which is the first line treatment for complicated malaria and uncomplicated malaria for children less than five kilograms body weight was stocked in $80 \%$ of the pharmacies. This is an indication that private community pharmacies in Dar es Salaam complement public health facility services.

Drug dispensers had low to medium knowledge about antimalarial drug use in pediatrics. Interestingly, majority of drug dispensers found to be dispensing drugs in the pharmacies were not trained pharmaceutical personnel. Although pharmaceutical personnel were comparatively more knowledgeable than nonpharmaceutical personnel, none of the dispensers could be categorized as highly knowledgeable regarding dispensing and use of antimalarial drugs in paedistrics.

Lack of knowledge of antimalarial drug use in pediatrics is a serious problem, especially in areas of intense transmission such as Dar es Salaam, where antimalarial drugs are given repeatedly to treat frequent fevers (even in the absence of malaria), thus increasing the risk of resistance and adverse drug reactions [11]. Although majority of drug dispensers knew that artemether-lumefantrine was the drug of choice for treatment of uncomplicated malaria, $61 \%$ were willing to dispense antimalarial drugs to pediatrics in the absence of a prescription. Only $47 \%$ of those who were willing to dispense antimalarial drugs without a prescription requested to know the weight of the child before dispensing the drugs. These results show that there is irrational dispensing of antimalarial drugs in pediatrics, which may lead to under- or over-dosing of patients, leading to drug resistance or toxic effects.

A similar study that was conducted in Dar es Salaam revealed that drug dispensers in private pharmacies had poor knowledge in providing accurate information to patients on the dosage of the new antimalarials and likely side/adverse effects as well as instructions on how to take the medicines [12]. These findings and the results of this study are in agreement with the fact that drug dispensers working in the private retail pharmacies were not trained on the new antimalarial treatment guidelines before the antimalarial policy changes were implemented in 2006 in Tanzania.

A recent cross-sectional community survey conducted in Tanzania showed that adults would be treated more frequently with shop bought drugs, while children were more often brought to a health facility [13]. In these health facilities, both prescribers and drug dispensers are expected to provide medication information to patients, especially when drugs are intended for pediatric use. In this study, we assessed the level of understanding of the medication information given by prescribers to the parents at the public health facilities regarding antimalarial drug use in pediatrics. Majority of respondents were young parents in the age group of 20-29 years of age. Most of these parents had also completed primary level 
education. It was therefore expected that instructions given by prescribers regarding drug use in pediatrics would easily be understood by parents. In general, parents had low to medium level of understanding the instructions. This raises a question on whether the information about prescribed antimalarial drugs was offered voluntarily by prescribers, or patients were expected to ask questions. Dar es Salaam being endemic for malaria, provision and understanding of the instructions about use of antimalarial drugs is necessary for parents to be able to care for children affected by malaria at home.

Due to high numbers of patients attended to by the prescribers at the public hospitals, it is difficult to weigh every child before prescribing antimalarial drugs. As a result, prescribers rely on the information given by parents about the weight of the children. Lack of ascertaining the weight of a child is likely to result in prescribing wrong dosage or wrong antimalarial drug to a child. This was evident by the observation that $35.5 \%$ of the children were prescribed quinine despite the fact that they all had $>5 \mathrm{~kg}$ of weight, did not present with any signs and symptoms of severe malaria or contraindication to artemetherlumefantrine. This could have a negative impact on patients' satisfaction with the care provided in public health facilities, resulting in self-medication as many patients may skip doctor consultations and go straight to private pharmacies to obtain their medicines [14].

In this study, apart from antimalarial drugs, children were also prescribed with cotrimoxazole and amoxicillin. The use of antibiotics in this study is much lower compared to the reports from other studies $(4,15)$. The misuse of antibiotics is often due to uncertainty about the diagnosis; as the clinical picture may not be clear. The recent introduction of Malaria Rapid Diagnostic Tests (MRDT) in Tanzania has dramatically improved diagnosis of malaria. The current use of MRDT may have contributed to accurate diagnosis of malaria and hence low uses of antibiotics seen in this study.

\section{Limitations of the study}

This study was conducted in Dar es Salaam, which is the commercial capital of Tanzania with high numbers of community pharmacies and prescribers in public hospitals. Therefore, the knowledge and practice of health care providers reported in this study may be at a higher level than in rural areas. In addition, a substantial number of children with suspected malaria are often treated at health centers and dispensaries in Dar es Salaam [13]. These facilities are often faced with shortages of qualified prescribers and drug dispensers. It is most likely that gaps in knowledge among health care personnel at these lower-tier health facilities are much greater than in hospitals. It is therefore recommended that a similar and bigger study be conducted to include different levels of health facilities in urban and rural areas.

\section{CONCLUSION}

This study has revealed irrational dispensing, prescribing and subsequently inappropriate use of antimalarial drugs in pediatrics. Due to the fact that anti-malarial drug policy was recently changed in Tanzania, it is suggested that interventions such as developing and circulating easy-to-use treatment guidelines and a regular audit of the application of these guidelines be instituted. In addition, continuing medical and pharmaceutical education of prescribers and drug dispensers on rational drug use and evidence-based medicine should also be instituted.

\section{ACKNOWLEDGEMENT}

We thank the medical officers in-charge of Dar es Salaam municipal hospitals for granting permission and support to conduct the study. We acknowledge the assistance by the Tanzania Food and Drugs Authority for providing a list of retail private community pharmacies in Dar es Salaam. Parents of children seeking for malaria treatment at municipal hospitals are acknowledged for their willingness to participate in the study. 
We thank the prescribers for providing responses to study questions.

\section{REFERENCES}

1. Ministry of Health Government of Tanzania. National Malaria Control Program; National Malaria Medium Term Strategic Plan, 20032007. Dar es Salaam, Tanzania: Ministry of Health; 2003.

2. Jowett M, Miller NJ. The financial burden of malaria in Tanzania: implications for future government policy. Int J Health Plann Manage 2005; 20: 67-84.

3. Oshikoya KA, Chukwura HA, Ojo 1O. Evaluation of outpatient pediatric drug prescriptions in a teaching hospital in Nigeria for rational prescription. Paediatr Perinat Drug Ther 2006; 7: 183-188.

4. Nwolisa CE, Erinaugha EU, Ofoleta SI. Prescribing practices of doctors attending to under-fives in a children's outpatient clinic in Owerri, Nigeria. $J$ Trop Pediatr 2006; 52: 197-200.

5. Goodman C, Kachur SP, Abdulla S, Mwageni E, Nyoni J, Schellenberg JA, Mills A, Bloland P. Retail supply of malaria-related drugs in rural Tanzania: risks and opportunities. Trop Med Int Health 2004; 9: 655-663.

6. Abuya TO, Mutemi W, Karisa B, Ochola SA, Fegan $G$, Marsh V. Use of over-the-counter malaria medicines in children and adults in three districts in Kenya: Implications for private medicine retailer interventions. Malar J 2007; 6: 57.

7. Goel $P$, Ross-Degnan D, Berman P, Soumerai $S$. Retail pharmacies in developing countries: a behavior and intervention framework. Soc Sci Med 1996; 42: 1155-1161.
8. Stewart $D$, Rouf $A$, Snaith A, Elliott $K$, Helms PJ, McLay JS. Attitudes and experiences of community pharmacists towards pediatric offlabel prescribing: a prospective survey. $\mathrm{Br} J$ Clin Pharmacol 2007; 64: 90-95.

9. Kachur SP, Abdulla S, Goodman C. Putting the genie back in the bottle? Availability and presentation of oral artemisinin compounds at retail pharmacies in urban Dare $s$ Salaam. Malaria J 2006; 5: 25.

10. Nyazema $N$, Viberg $N$, Khoza $S$, Vyas $S$, Kumaranayake L, Tomson G, Lundborg CS. Low sale of antibiotics without prescription: a cross-sectional study in Zimbabwean private pharmacies. J Antimicrob Chemother 2007; 59: 718-726.

11. Whitty CJ, Rowland M, Sanderson F, Mutabingwa TK. Malaria. BMJ 2002; 325: 1221-1224.

12. Minzi OMS, Haule AF. Poor knowledge on new malaria treatment guidelines among drug dispensers in private pharmacies in Tanzania: The need for involving the private sector in policy preparations and implementation. East Afr J Pub Health 2008; 5: 117-121.

13. Hetzel MW, Obrist B, Lengeler C, Msechu JJ, Nathan $R$, Dillip A, Makemba A, Mshana $C$, Schulze A, Mshinda $H$. Obstacles to prompt and effective malaria treatment lead to low community-coverage in two rural districts of Tanzania. BMC Public Health 2008; 8: 317.

14. Mamdani M, Bangser M. Poor people's experiences of health services in Tanzania: a literature review. Reprod Health Matters 2004; 12: 138153.

15. Mannan AA, Malik EM, Ali KM. Antimalarial prescribing and dispensing in health centers of Khartoum state, 2003-04. East Mediterr Health J 2009; 15: 122-128. 